\title{
Software Composition Using Behavioral Models of Design Patterns
}

\author{
Sargon Hasso ${ }^{1}$, Carl Robert Carlson ${ }^{2}$ \\ ${ }^{1}$ Technical Product Development, Wolters Kluwer Law and Business, Chicago, USA; ${ }^{2}$ Information Technology and Management, \\ Illinois Institute of Technology, Wheaton, USA. \\ Email: sargon.hasso@wolterskluwer.com
}

Received January $1^{\text {st }}, 2014$; revised January $30^{\text {th }}, 2014$; accepted February $7^{\text {th }}, 2014$

Copyright (C) 2014 Sargon Hasso, Carl Robert Carlson. This is an open access article distributed under the Creative Commons Attribution License, which permits unrestricted use, distribution, and reproduction in any medium, provided the original work is properly cited. In accordance of the Creative Commons Attribution License all Copyrights (C) 2014 are reserved for SCIRP and the owner of the intellectual property Sargon Hasso, Carl Robert Carlson. All Copyright @ 2014 are guarded by law and by SCIRP as a guardian.

\section{ABSTRACT}

Given a set of requirements structured as design problems, we can apply design patterns to solve each problem individually. Much of the published literature on design patterns addresses this problem-pattern association; however, there is no systematic and practical way that shows how to integrate those individual solutions together. We propose a compositional model based on design patterns by abstracting their behavioral model using role modeling constructs. This approach describes how to transform a design pattern into a role model that can be used to assemble a software application. The role model captures the behavioral relationship between participant components in the design pattern. Our approach offers a complete practical design and implementation strategies, adapted from DCI (Data, Context, and Interaction) architecture. We demonstrate our technique by presenting a simple case study complete with design and implementation code. We also present a simple to follow process that provides guidelines of what to do and how to do it.

\section{KEYWORDS}

Software Composition; Design Patterns; Integration; Role Model; Architecture; DCI Architecture; System Responsibilities; Traits

\section{Introduction}

In our prior research [1], we laid out the foundational theory for constructing system architecture by composing components using design patterns [2] as solutions to integration problems. The use of patterns as integration mechanism is different from using them, as originally conceived, as solutions to design problems. Integration based on design patterns, as we will show later, is behavioral in nature, i.e. based on collaboration, and is semantically richer than the traditional structural-based approach using generalization, aggregation, and association. The literature abounds with techniques to help designers practice and apply design patterns in building applications; however, very little attention is paid to how to assemble applications in a systematic way from patternbased components.

In examining how the Lexi editor case study was as- sembled in Gamma et al. [2] book, or how the hierarchical file system (HFS) case study was assembled in Vlissides [3] book, it is not very obvious how the final application is assembled from components without explaining the assembly, or composition, process explicitly. From our teaching experience to students who are assigned design projects to build applications using design patterns, similar to Lexi and HFS, we found out that they struggle with integrating components together. This problem motivated us to research this problem and come up with an approach to integrate components using design patterns themselves as an abstraction mechanism and transforming those abstractions into realization during implementation. To emphasize, we are introducing design patterns as abstract modeling elements to solve concrete software composition problems.

Here is how this paper is organized. In Section 2, we briefly survey the current design patterns-based tech- 
niques for software composition. In Section 3, we lay out the conceptual background needed to use our approach. In Section 4, we describe just enough concepts from DCI architecture we need for our implementation strategy. To provide support in following our proposed approach, we present in Section 5 a simple to follow process that provides guidelines of what to do and how to do it. A simple case study is introduced in Section 6 demonstrating our approach during design and implementation. A brief discussion is given in Section 7, then our conclusion and future work are discussed in Section 8. To make the concepts concrete, we also provide a complete source code listing of the case study in Appendix.

\section{Related Work}

Decomposing an application into design problems and finding solutions based on design patterns creates an integration problem designers must deal with. This is also true even though design solutions are not patterns-based. Bass et al. [4] talk about one of the desired system quality attributes a software architecture should have, namely integrability which they define as: "the ease with which separately developed components, including those developed by third parties, can be made to work together to fulfill the software's requirements”. Currently, there are no systematic approaches to integrate patterns-based components. Case studies found in Gamma et al. [2], and Vlissides [3] use ad hoc approaches to do integration. Moreover, the integration tends to be untraceable, unmethodical, order-dependent, and non-repeatable. It does rely heavily on the experience of designers to come up with integration strategies.

An approach by Yacoub et al. [5] uses design patterns for composition and those patterns are referred to as constructional design patterns. Basically these are design patterns plus an interface specification. Gluing patterns together is accomplished by two types of interfaces: classes and operations. In other words, two patterns can be integrated using either a shared object or an operation. The selection of either interfaces is arbitrary. The chosen object or operation comes from existing model elements in design patterns. The biggest disadvantage of this approach is the fact that you must, somehow, identify parts, either objects or operations, of the two patterns to be used as interfaces. If one pattern-based component requires an operation that a participating object from another pattern-based component does not have, this approach may not work.

Riehle [6] describes an approach for composing design patterns-based components using the roles concept. This approach still relies on roles' relationship similar to class' relationship. This is an unnecessary constraint during analysis phase. Furthermore, his composition technique constrains pattern integration to produce composite designs that are patterns themselves which limits the wide applicability of this approach.

Our approach offers a complete design and implementation strategies with a set of techniques that most software engineers are familiar with. Contrast this approach with the formal approaches we surveyed in the literature that are difficult to comprehend and implement unless proper software tools are available. For example, the method in [7] starts with the explicit design pattern model structure as a basis for composing patterns by specifying their structural and behavioral properties using two types of logics: first-order logic [8] and temporal logic of actions [9], respectively. The resulting specifications are incomprehensible to most practitioners unfamiliar with formal methods of specifying designs.

\section{Theory: Conceptual Foundation}

Design patterns are commonly used as techniques that offer solutions to commonly recurring problems when building software components or applications [2]. However, we have come up with a compositional model based on design patterns by abstracting their behavioral model using role modeling constructs. What we mean by compositional model is similar to what we do when we assemble a software component from, say, two objects through typical software composition techniques like generalization, aggregation, and association. Shared object is another technique used for this purpose [2]. However, the compositional model exhibited by these techniques is structural. Naturally, this structure results when system functionality is decomposed into modules arranged into any number of possible arrangements. Our compositional model, on the other hand, is behavioral in nature because it is based on the collaboration model derived from design patterns that has specific semantics based on the design pattern we use. In order to describe this collaboration model, as we will illustrate shortly, we have to specify the design patterns as role models. Each design pattern we choose will have a different role model. How do we obtain these role models? For each design pattern, we examine its participants' collaboration behavior, and factor out their responsibilities. A responsibility is collection of behaviors, or functions, or tasks, or services. We then specify the resulting role model much like a collaboration model in UML [10] where it states that "roles in collaborations will often be typed as interfaces and will then prescribe properties that the participating instances must exhibit, but will not determine what class will implement those behavioral properties”. The resulting collaboration model will play the same function as a use case function in the DCI architecture [11] whose techniques we want to use to implement, in code, the 
integration process. It is very important to realize that in addition to using design patterns to solve a design problem, we are also proposing using design patterns to solve an integration problem. The fact that a design pattern has a collaboration context with participants with prescribed behavior is what we are abstracting.

In role modeling, each distinct system activity or a behavior, a use case for example, is considered and modeled individually. We generally examine the roles of two or more interacting entities during behavior analysis. The same entities may assume different roles in yet other interaction scenarios describing a different aspect of system behavior. In general, one system functionality may span several objects belonging to different classes (this is the same as saying that several objects, in their different roles, are collaborating to execute a function). Another system functionality may span the same or additional objects. However, this time the same objects may take on a different role. To describe a complete behavior of one specific object, the different roles are composed or synthesized. This resultant synthesized behavior is assembled and implemented as a class. It is highly likely that this role modeling is happening implicitly in the software designer's mind but the thought process can be made explicit and there are several approaches in the literature dealing with this problem.

Role diagrams that depict the role model is appropriate at this level of analysis because they involve the collaboration of two or more objects. This also provides the context to model the structure of object interaction [12]. This idea does not seem to be different from the way design patterns are defined: “... design pattern identifies the participating classes and instances, their roles and collaborations, and their distribution of responsibilities ...” [2].

Role modeling in this discussion, therefore, is used in two different ways: first, as a way to expose different interfaces by the same object, depending how it interacts with other objects, and second, as a way to describe collaboration between two or more objects during an enactment of, say, one system functionality or one use case scenario. The former is what traits [13] were used for, something we are not interested in here; while the latter is what we will be utilizing to model system behavior that is factorable. We will utilize the concept of a role as a partial description of an object's specifications during collaboration with other objects. Henceforth, when discussing design pattern components (participants), we will refer to them as illustrated in Figure 1(b). Essentially, this means the design pattern, in this case the Decorator [2] (p. 175), see Figure 1(a), has two components represented by two roles: Decorator and Component. It's these two roles that really get mapped or injected into objects when doing design integration using design patterns. As the diagram in Figure 1(b) shows, we use the UML's [10] collaboration as a dashed ellipse icon which represents the design pattern we are using as an integrator. In the collaborations model, we capture how a collection of communicating objects collectively accomplishes a specific task. We achieve composition by the virtue of how participants in the chosen design pattern communicate. The parts in each collaboration composite structure represent the roles that we factored out from each design pattern as an abstraction that ultimately need to be bound to objects from the integrated components as illustrated conceptually in Figure 2. The interface realizations in the diagram are necessary for statically typed languages.

Figure 3 illustrates, in a more concrete way, how certain behavior is factored out and packaged as a role mo$d e l$, see Figure 3(a). Then, as depicted in Figure 3(b), if we were given two components and we wish to integrate them in a manner similar to the behavior encapsulated by

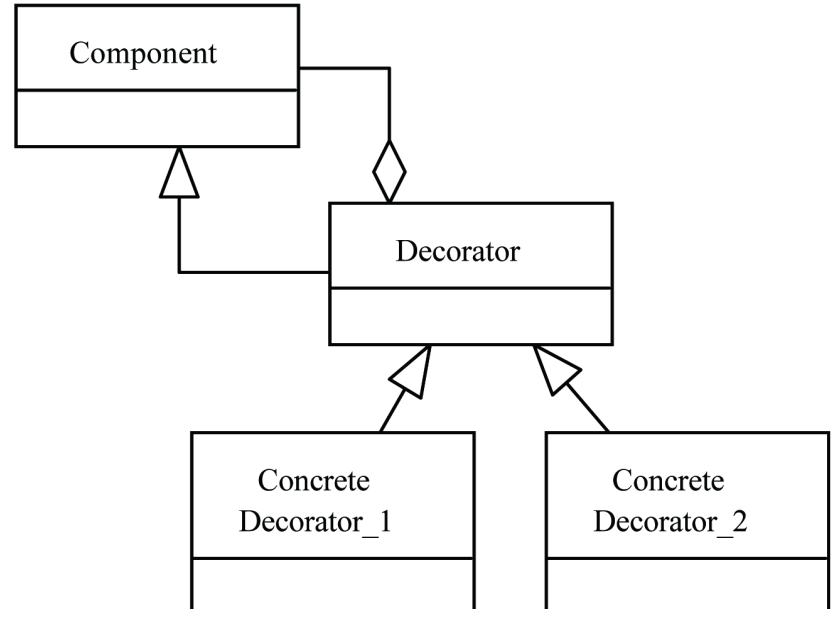

(a) Decorator Class Model

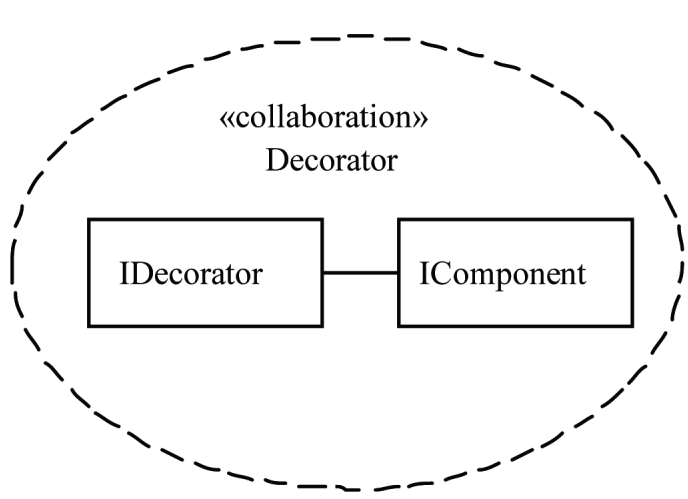

(b) Decorator Role Model

Figure 1. Illustration of the abstraction process from class model to collaboration or role model. 


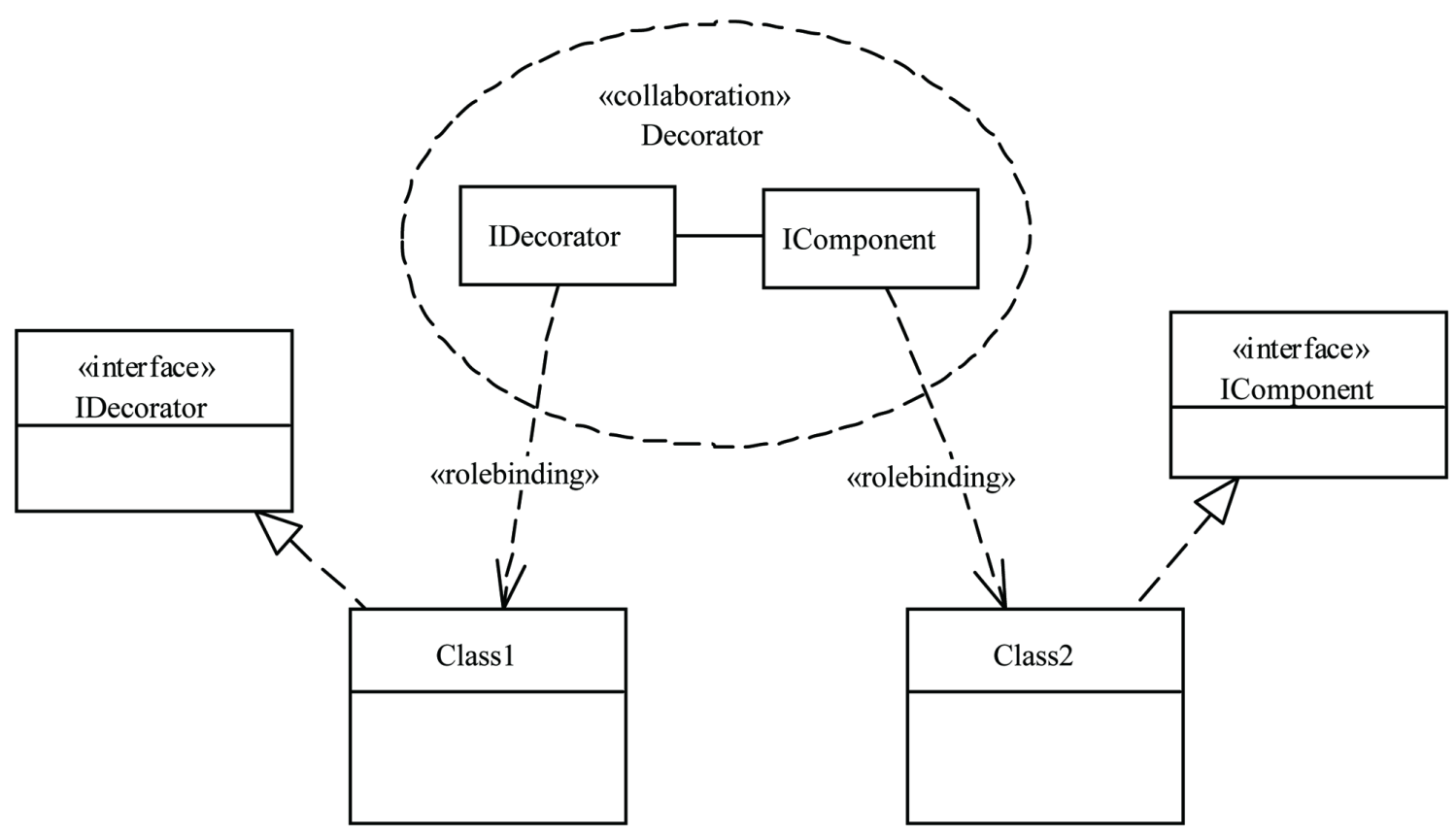

Figure 2. The role mapping process to arbitrary class instances.

the role model, we can pick out two objects, in this case C and G, and map roles IA and IB onto them, respectively. Since, the role model constitutes one specific collaboration to accomplish a certain task that involves two objects, the new objects that assume those roles will collaborate in similar manner. Therefore, by the virtue of this collaboration, we were able to combine (integrate) Component1 and Component2. This will be become more evident as we go through a detailed example in Section 6. As a stylistic convention, we prefix a role name with a letter "I" to denote an "interface" in code.

\section{Practice: DCI Architecture}

We briefly discuss the DCI architecture and show how we adapted it to implement our compositional model. The DCI architecture was introduced by Reenskaug [11] and further elaborated on extensively by Coplien et al. [14].

In DCI, we start with the use case model as a driving force to implement an application. The architecture of an application comprises the Data part, this describes the makeup of the system, and the Interaction part, this describes system's functionality. What connects the two dynamically is a third element called Context. Each of these three parts has physical manifestation as components during implementation. For example, there are objects to represent the applications' domain objects; objects to represent system behavior or interactions between domain objects; and objects to represent use cases. The architecture is clean in that it makes a clear distinction between design activities corresponding to each of the artifacts, namely the Data, Context, and Interaction. It also makes traceability between what the user wants and where it is implemented in the code clear through the use case context construct in the architecture.

The domain objects behavioral specification is highly cohesive by making each object knows everything about its state and how to maintain it. Coplien et al. [14] refer to these domain objects as dumb objects that know nothing about other objects in the system. The interaction between domain objects, on the other hand, is a system functionality captured as system behavior and assigned to yet another type of objects conveniently named as interaction objects. The DCI treats these objects as first class citizens. While the identification of domain object responsibilities, i.e. object behavior, is a technique known from early days of object oriented analysis and design, check for example Wirfs-Brock et al. [15] and Coad et al. [16] who refer to this task as "Do it Myself" strategy, the interaction between objects having its own object designation is a novel concept the DCI re-introduced and made it a visible modeling element in system architecture.

In DCI architecture, systems provides hints to system responsibilities with respect to use cases. In a typical use case scenario, system entities interact with each other through defined roles. These roles, ultimately, will be mapped onto domain objects instantiated at runtime. The DCI elaborates on this process-but all we care about at design time is identification of those object roles and what kind of behavior is expected of them. Therefore, object interactions are use case enactments at runtime. 


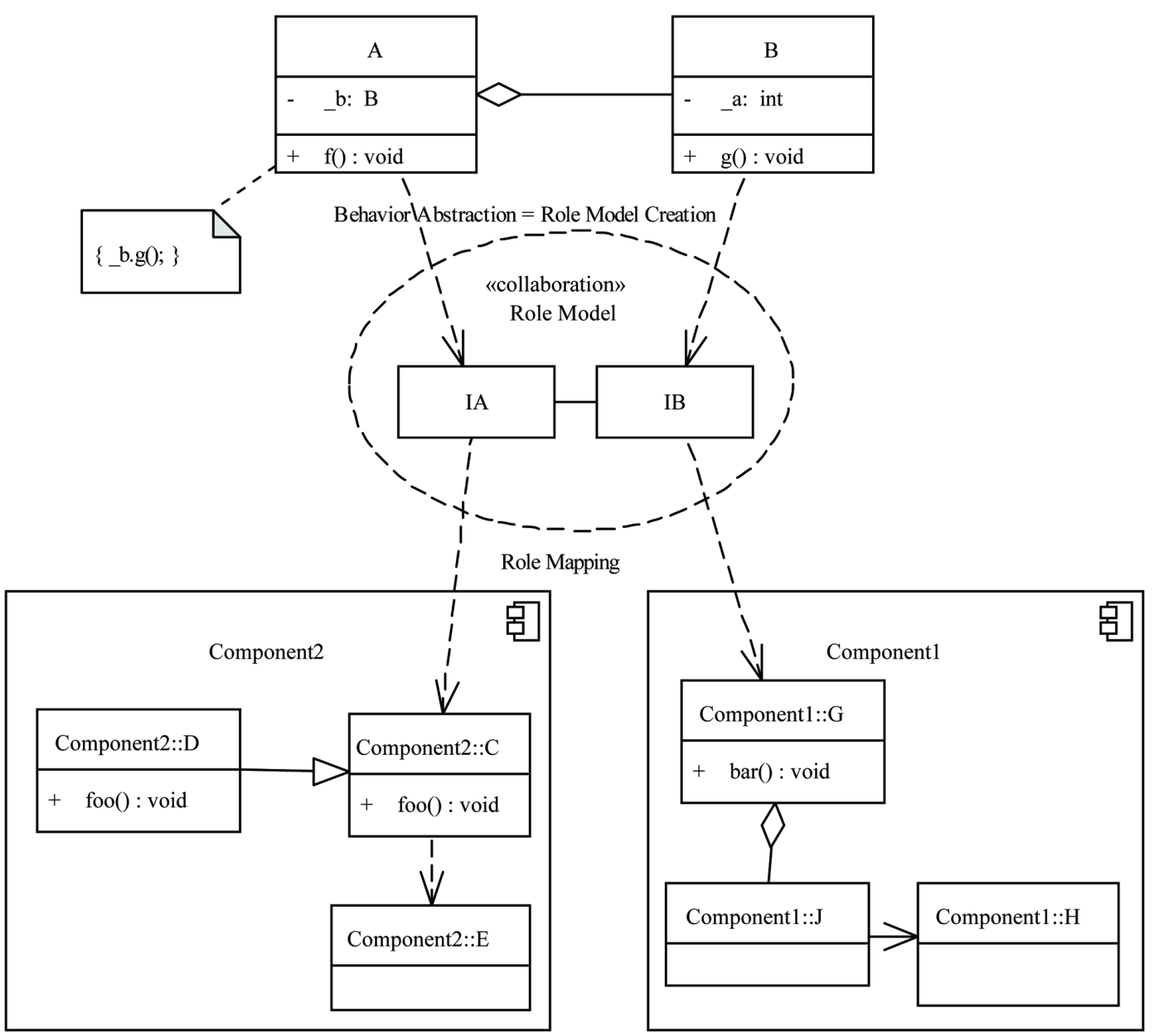

(a) Behavior Abstraction and its Corresponding Role Model

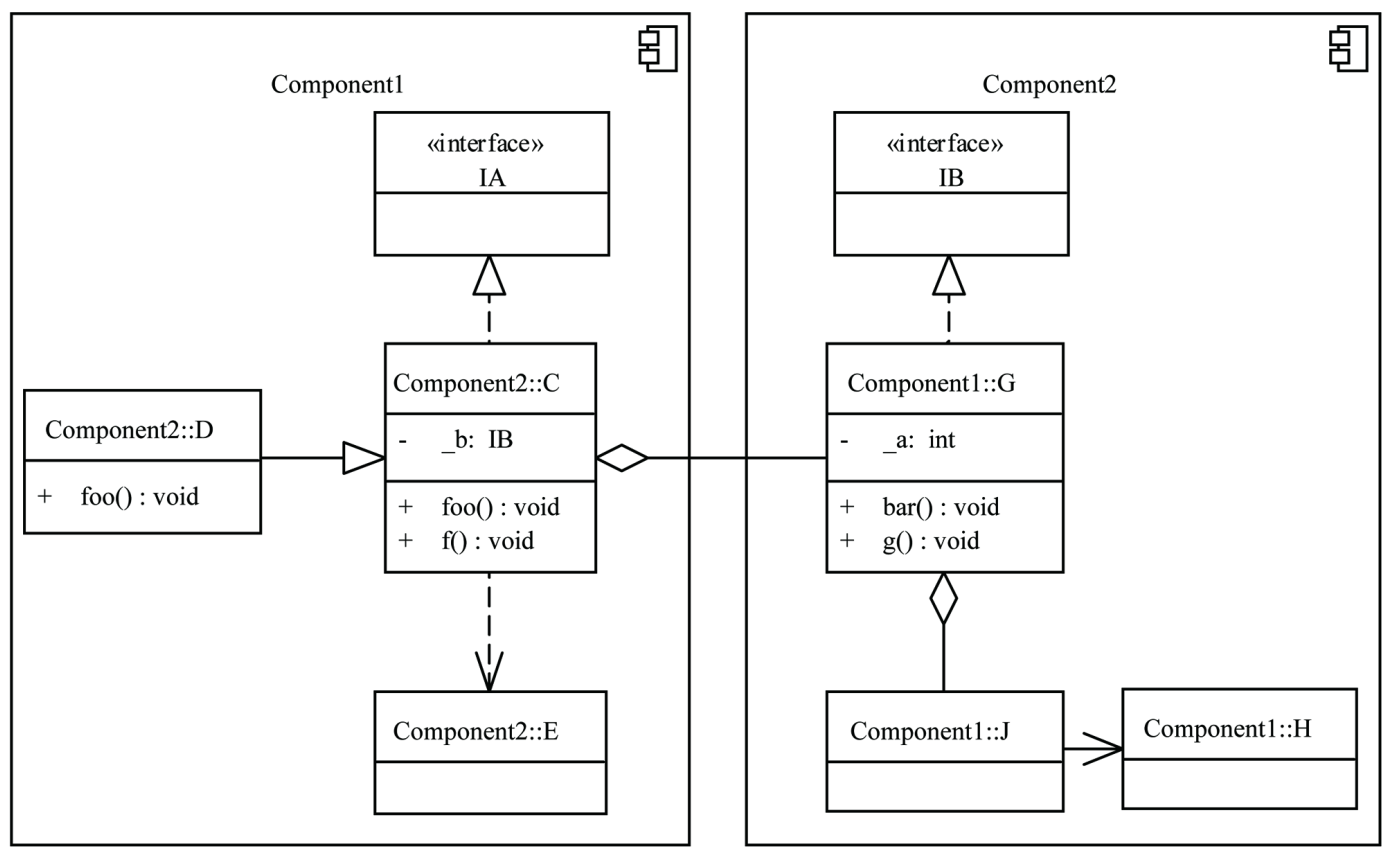

(b) Role Model as a Reusable Construct and its Component Integration as a side effect

Figure 3. Role model abstraction and integration through mapping. 
System functionality, i.e. functionality that does not belong to any one specific object type at design time, is injected onto roles at runtime and when any object plays that role, i.e. it has acquired a new behavior. This is accomplished using a programming construct called Traits first introduced by Schärli et al. [13] and is defined as "a group of methods, i.e. behavior, that serves as a building block for classes and is a primitive unit of code reuse."

\section{A Software Composition Process Using Design Patterns}

After covering theory and practical implementation strategy, we present the following process by which we use design patterns in their role specification as new means to integrate components. The key concepts and core ideas we borrowed from DCI architecture and adapted them for our process are: role specifications, behavior injection through "traits mechanism", i.e. extending the functionality of any object, and introducing a collaboration context similar to use case context.

1) Design each component with all the required functionality. We realize that interdependencies on services from other components are required; therefore, we assume that it may be necessary to introduce an architectural layer that provides the necessary abstraction level.

2) Determine the requirements needed for two components to interact. This step specifies the collaboration between the components.

3) Select one design pattern that may satisfy this requirement.

4) Identify design patterns' participant roles.

5) Code up the roles as methodless interfaces; however, some roles may contain other roles as properties.

6) Identify the responsibility of each role and code it up as a Trait.

7) Select an object from each component that we need to map each role onto.

8) Map the design pattern participants' roles to these objects. The implementation is language dependent, but for statically typed languages Interface-like implementation is common.

9) Create a context class for the collaboration to take place identified in Step 2.

\section{Case Study: A Library System}

We will illustrate our approach, and follow our process along the way, using a case study that we intentionally made it simple to focus on key concepts presented in this paper. We state few requirements, design a solution, and provide a complete implementation in Appendix. We numbered the code listing for easy reference. This system supports these requirements:
1) A local library has library services, resource collections, and administration offices.

2) A local library system uses services of a remote lending branch.

3) Library services are either simple services (books reservation, DVDs reservation, CDs reservation, and search services) or composite services.

4) Resource reservations are made by any user.

5) Users can search for resources.

6) Loanable resources, e.g. books, DVDs, and CDs, are either available or checked out.

The application is decomposed into three distinct components depicted by component diagrams in Figures 4(a)-(c) corresponding to our three structural requirements 1, 6, and 3 listed above, respectively. The intent is to integrate these three components using our proposed approach based on design patterns. The integration requirement comes from requirement 4 and 5 (Step 1).

Figure 5 illustrates how we intend to integrate the three components using the Proxy [2] (p. 207) and State [2] (p. 305) design patterns. We use the Proxy design pattern as an integrator because the Library Services relies on remote services from a lending branch. Needless to say, this is a contrived example to demonstrate the technique. By similar reasoning, we opted to use the State pattern as an integrator between the Lending Branch Books Reservation Services and Loanable Resources, i.e. books, components (Steps 2 and 3). In Figure 5, we show two collaboration models corresponding to Proxy and State patterns that we will use as integrators in our case study. We will show how to code up these structures using C\# language. We only describe integrating two components using the Proxy pattern; however, the process is exactly similar to integrating the other components using the State pattern (Step 4). In the code, lines 11-17, these roles are implemented as methodless interfaces (Step 5):

\section{public interface ISubject \{\} \\ public interface IProxy \{\}}

In the code, lines 22 and 44, two objects, Services from the Library component and LendingBranchServices from the Lending Branch Services component, will implement IProxy and ISubject interfaces, i.e. roles, respectively (Steps 7 and 8):

$$
\begin{aligned}
& \text { public class Services: IProxy }\{\} \\
& \text { public class LendingBranchServices: ISubject }\{\}
\end{aligned}
$$

Of course, there is nothing to implement since these are methodless interfaces. Based on the DCI architecture strategy, they serve as identifiers for objects that will take those roles. The Proxy design pattern, basically, is a stand-in for another object. The target Search() or CheckOut() methods will be called by a Request method that 


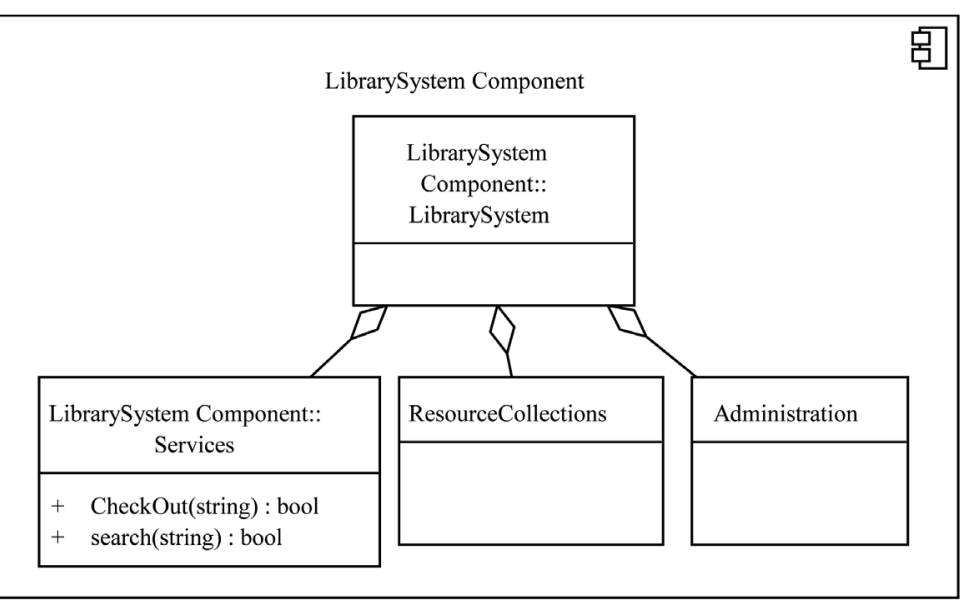

(a) Library System Component

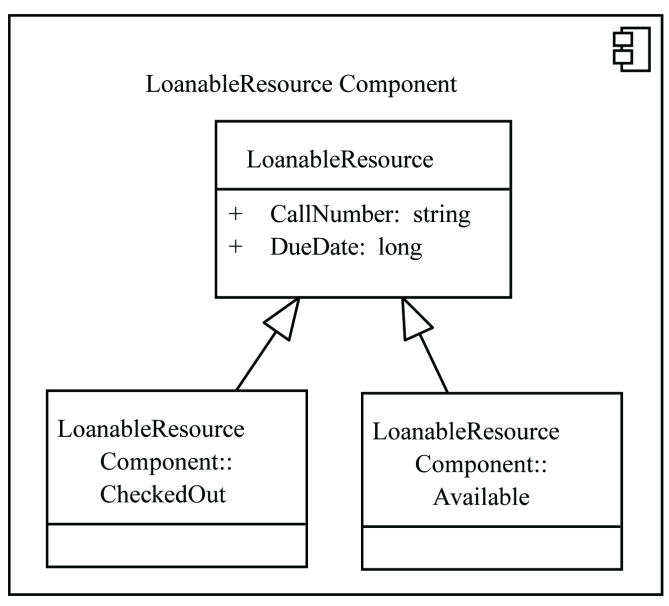

(b) Loanable Resource Status Component

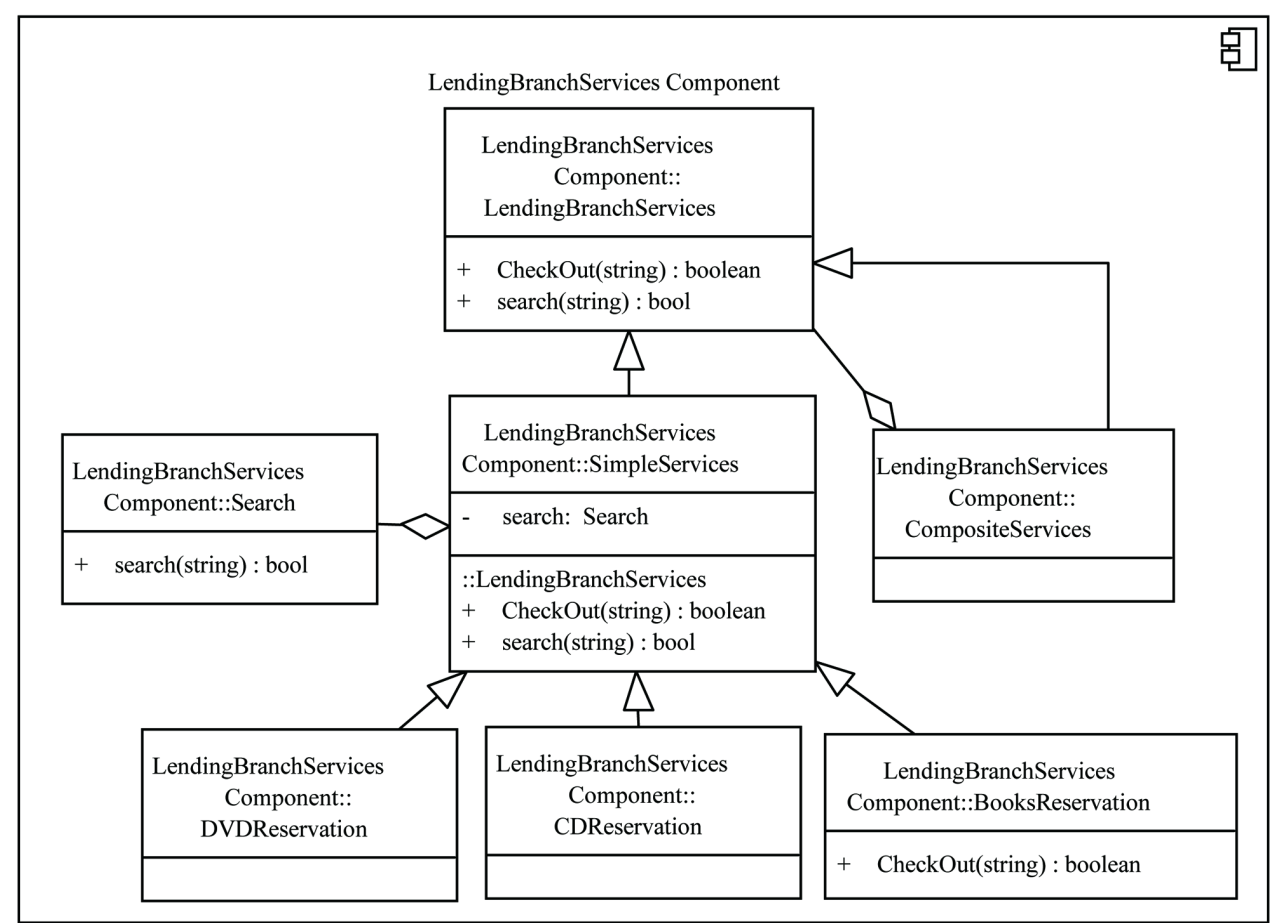

(c) Lending Branch Services Component

Figure 4. Model structure of the three individual components of the library system sample application.

we will be injecting into IProxy type object by the Trait [13] concept. In C\# language, it is done through extension method [17]. Extension methods enable you to "add" methods to existing types without creating a new derived type, recompiling, or otherwise modifying the original type. Extension methods are a special kind of static method, but they are called as if they were instance methods on the extended type. This is what we did in the RequestTrait class (Step 6) line 103 in the code.

public static class RequestTrait \{\} public static bool Request (this IProxy proxy, ISubject subject,

\section{$\ldots\}$ \\ Request Type request) \{\}}

In fact this class contains the behavior associated with IProxy role that gets injected into any object taking this role, e.g. objects of class Services. In C\# a Request() method extends, i.e. adds more methods, any arbitrary object with new behavior as long as it is of type IProxy in our case. This is done through the first argument of Request(this IProxy proxy,...) method.

The last piece of the puzzle to make all this work is the integration. We create a context that corresponds to the "collaboration” that acts as integrator. This is similar to 


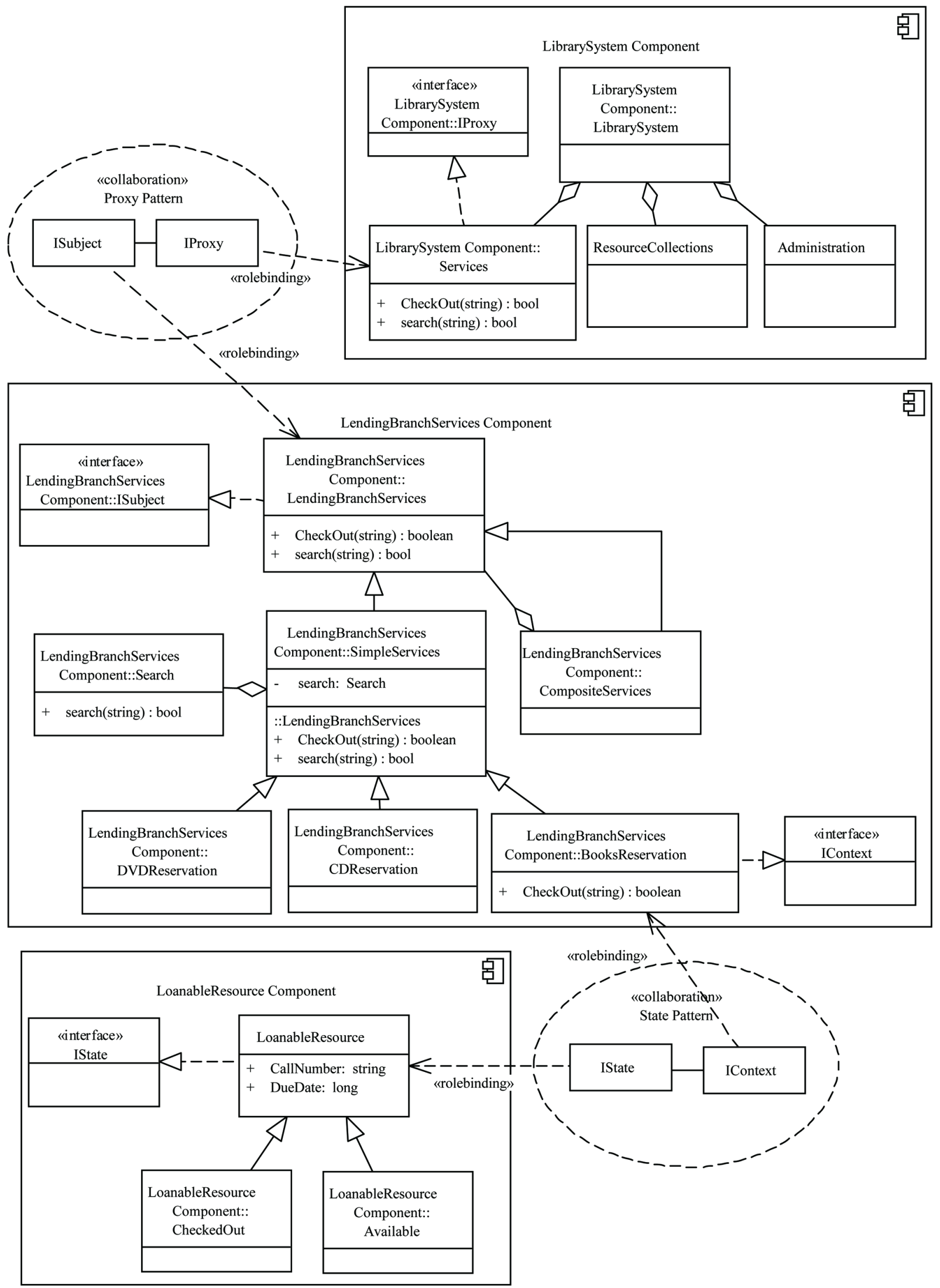

Figure 5. Library system consisting of three components integrated using design patterns. 
how the DCI architecture creates a "context" class for each use case. The RequestResourceContext class is the place for this to happen (Step 9) line 153 in the code.

\section{public class RequestResourceContext \{\}}

As you can see, the "integration" which is based on the "collaboration" model is a construct that is quite traceable in the code. The integration happens when we instantiate an object of type "RequestResourceContext", line 195, after setting up its required parts (through its constructor) and calling its "Doit" method in the Main method of the LibrarySystemCaseStudy class, line 196. Now, you see why we call this type of integration behavioral since it is based on a method call at runtime. Using the State pattern adds a slight complication because the IContext requires IState property, lines 13-16. However, this property is of the type getter and setter whose code is easily generated by most modern interactive development environments. In the code, we demonstrate how a loanable resource, i.e. a book, started in Available state, line 194, checked out, line 196, and became available again, line 197. In the code, lines 198-199, we also demonstrate how the Search() method that was injected through IProxy role, is invoked through the integration mechanism between Services and LendingBranchServices objects.

Figure 6 is a high-level view of the components assembly showing the design patterns as the integration interfaces representing the wiring of the three components.

We left out some of the detailed explanation of the rational behind using Traits and Methodless roles and some of the limitations of the statically typed languages, like

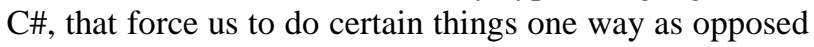
to dynamically typed languages where there is more flexibility of injecting a role at runtime rather than at compile time. Chapter 9 of Coplien et al. [14] has all this explained. The complete workable code, albeit skeletal, is listed in Appendix.

\section{Discussion}

On encountering our approach for the first time, one may get the impression it is no different from Gamma's or Vlissides' approaches. There is, however, a subtle dif ference in that our approach provides explicit steps to integrate software components. The technique can be applied repeatedly to any integration problem. First andforemost the approach presented in this research is of practical importance. The theory serves only to validate the concrete implementation and provides generalization to a variety of implementation strategies. The key concepts to take away are these. First, design patterns' key principal properties are used as abstraction modeling constructs through collaboration. These, then become traceable artifacts through "context" classes in the code. Second, the proposed approach allows for partial and evolutionary design. Recall, that the collaboration model captures all the integration requirements by the virtue of the role model it encapsulates. Third, role to object mapping is really a binding mechanism that could be utilized effectively by this duality principle: either domain objects discovery or object roles allocation can be deferred. In other words, you can begin design with domain objects if you have settled on all of them, or you can begin design with roles required behavior and then map or bind them to objects at a later time. The latter gives you the most flexibility. Last, we provide a process anyone can learn and follow methodically.

Could we have used a different pattern to integrate? Absolutely, and which one we choose depends on requirements. Let's say that the Search() method of Services class and the Search() method of the LendingBranchServices class had incompatible interfaces. In that case, we could use the Adapter pattern [2] (p. 139) whose participants have the roles of IAdpater and IAdaptee. The behavior of the Adapter role, i.e. adapting a generic Request to a Specific Request, would have been the trait class.

Is any design pattern suitable as an integrator? It depends on how well you structure your composition problem in such a way that matches the design problem a specific design pattern intends to solve. In addition to reuse, design patterns promote flexible designs; by the same argument one can use design patterns to create flexible architectural compositions. This is one of the characteristics of maintainability which is a desired design quality attribute.

The design and the implementation approach we presented creates a new design paradigm that appears complex at first but once learned, it becomes another powerful tool added to architect's and designer's skill set. The

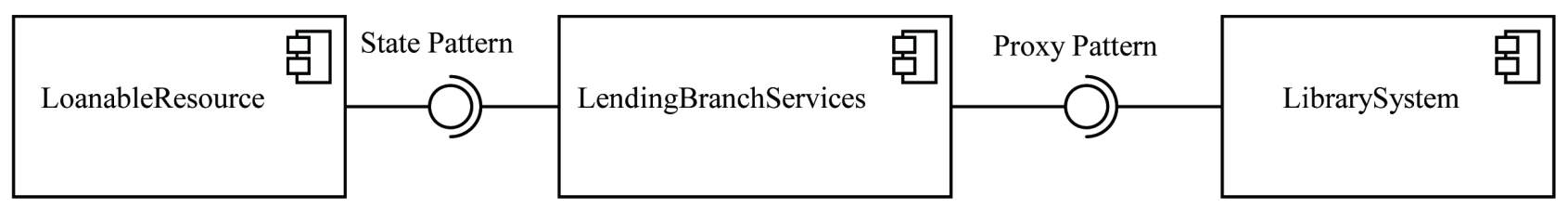

Figure 6. An abstract view of library system components assembly. 
compositional model requires creating abstractions out of behavioral collaboration models of design patterns. Although this type of integration has richer semantics, it is not as straight forward as using the traditional techniques like aggregation or generalization. It forces you to think and design in the abstract something not many feel comfortable with. Furthermore, since the implementation strategy follows, more or less, the DCI architecture footsteps, it also suffers from some of the added overhead introduced by that architecture, as discussed in Coplien et al. [14] (pp. 294-297).

\section{Conclusion and Future Work}

We have introduced a conceptual framework and an implementation model for software composition using design patterns. We have also created a process that should guide practitioners and first time learners, learning how to use design patterns, in assembling individual components. That is our contribution. The compositional model can also be used for non-pattern-based components. The approach is scalable without adding complexity and should work with any design pattern once its collaboration model is identified. The rational used to select a design pattern to solve design problems should also work for selecting a design pattern to solve integration problems.

For future research, there is an opportunity to automate some of the implementation tasks with proper code generators, e.g. metaprogramming techniques available in some development frameworks like .NET [18]. Code injection through Reflection could easily be accomplished at compile time. Also, since the approach allows defering the integration until a later stage in the development cycle, it gives an opportunity for architects or designers to identify a variation point, i.e. integration strategy, with variants [19].

Finally, to evaluate this proposed compositional model against other ad-hoc approaches, we intend to conduct a design experiment that is formal, rigorous, and controlled based on techniques from experiments in software engineering [20]. This effort is part of future work.

\section{Acknowledgements}

The open access support for this work was supported by the Illinois Institute of Technology.

\section{REFERENCES}

[1] S. Hasso, "A Uniform Approach to Software Patterns Classification and Software Composition,” Ph.D. Thesis, IIlinois Institute of Technology, 2007.

[2] E. Gamma, R. Helm, R. Johnson and J. Vlissides, "Design Patterns,” Addison Wesley, Reading, 1995.

[3] J. Vlissides, "Pattern Hatching: Design Patterns Applied of Software Patterns,” Addison Wesley, Reading, 1998.

[4] L. Bass, P. Clements and R. Kazman, "Software Architecture in Practice of SEI Series in Software Engineeering,” 2nd Edition, Addison-Wesley Professional, Boston, 2003.

[5] S. M. Yacoub and H. H. Ammar, "Pattern-Oriented Analysis and Design (POAD): A Structural Composition Approach to Glue Design Patterns," 34th International Conference on Technology of Object-Oriented Languages and Systems, Santa Barbara, 30 July-04 August 2000, pp. 273-282.

[6] D. Riehle, "Describing and Composing Patterns Using Role Diagrams," Proceedings of the 1996 Ubilab Conference, Zurich, 1996.

[7] T. Taibi, "Formalizing Design Patterns Composition," The IEE-Proceeding Software, Vol. 153, No. 3, 2006, pp. 127-136. http://dx.doi.org/10.1049/ip-sen:20050072

[8] H. B. Enderton, “A Mathematical Introduction to Logic,” Academic Press, 2nd Edition, 2000.

[9] L. Lamport, "The Temporal Logic of Actions," ACM Transactions on Programming Languages and Systems (TOPLAS), Vol. 16, No. 3, 1994, pp. 872-923. http://dx.doi.org/10.1145/177492.177726

[10] OMG, “OMG Unified Modeling Language (OMG UML), Superstructure,” 2.4.1 Edition, Object Management Group, 2011.

[11] T. Reenskaug and J. O. Coplien, "The DCI Architecture: A New Vision of Object-Oriented Programming,” 2009. http://www.artima.com/articles/dci visionP.html

[12] T. Reenskaug, "Working with Objects: The OOram Software Engineering Method,” Manning Publications, 1996.

[13] N. Schärli, S. Ducasse, O. Nierstrasz and A. P. Black, "Traits: Composable Units of Behaviour," Proceedings of European Conference on Object-Oriented Programming (ECOOP'03), Vol. 2743, pp. 248-274.

[14] J. Coplien and G. Bjørnvig, "Lean Architecture: For Agile Software Development," 1st Edition, Wiley, West Sussex, 2010.

[15] R. Wirfs-Brock and A. McKean, “Object Design: Roles, Responsibilities, and Collaborations,” Addison-Wesley, Boston, 2003.

[16] P. Coad, D. North and M. Mayfield, "Object Models: Strategies, Patterns, and Applications,” Yourdon Press, Upper Saddle River, 1997.

[17] Microsoft Corp, “C\# Programming Guide: Extension Methods,” 2012.

http://msdn.microsoft.com/en-us/library/vstudio/bb38397 7.aspx

[18] K. Hazzard and J. Bock, "Metaprogramming in .NET,” Manning Publications Co., Shelter Island, 2013.

[19] K. Phol, G. Böckle and F. van der Linden, "Software Product Line Engineering: Foundations, Principles, and Techniques,” Springer, Heidelberg, 2010.

[20] C. Wohlin, P. Runeson, M. Höst, M. C. Ohlsson, B. Regnell and A. Wesslén, "Experimentation in Software Engineering: An Introduction of the Kluwer International Series in Software Enginerring,” Kluwer Academic Pub- 
lishers, Boston, 2000.

http://dx.doi.org/10.1007/978-1-4615-4625-2

[21] S. Hasso, "Design Patterns as Connectors Source Code on
GitHub,” 2014

https://github.com/shasso/LibrarySystemJSEA2014 


\section{Appendix}

This is a complete skeletal code listing in C\# language. Please refer to Section 6 for discussion and Figure 5 for the class model of the three components we are composing to make up the final application. The complete Visual Studio solution can be downloaded from GitHub [21].

1 using System;

2 using System.Collections.Generic;

3 using System.Linq;

4 using System.Text;

5

6 namespace Roles

7\{

8

9

// role declaration: a place holder with methods behavior)

10 // willing to take this role

11 public interface ISubject \{\}

12 public interface IProxy \{\}

13 public interface IContext

14\{

$18\}$

19 namespace LibrarySystem

20\{

21 using Roles;

\section{CDReservation,}

public enum RequestType

39 \}

40 namespace LendingBranchServices

41\{

42 using Roles;
43 using LibrarySystem;

44 public class LendingBranchServices : ISubject 


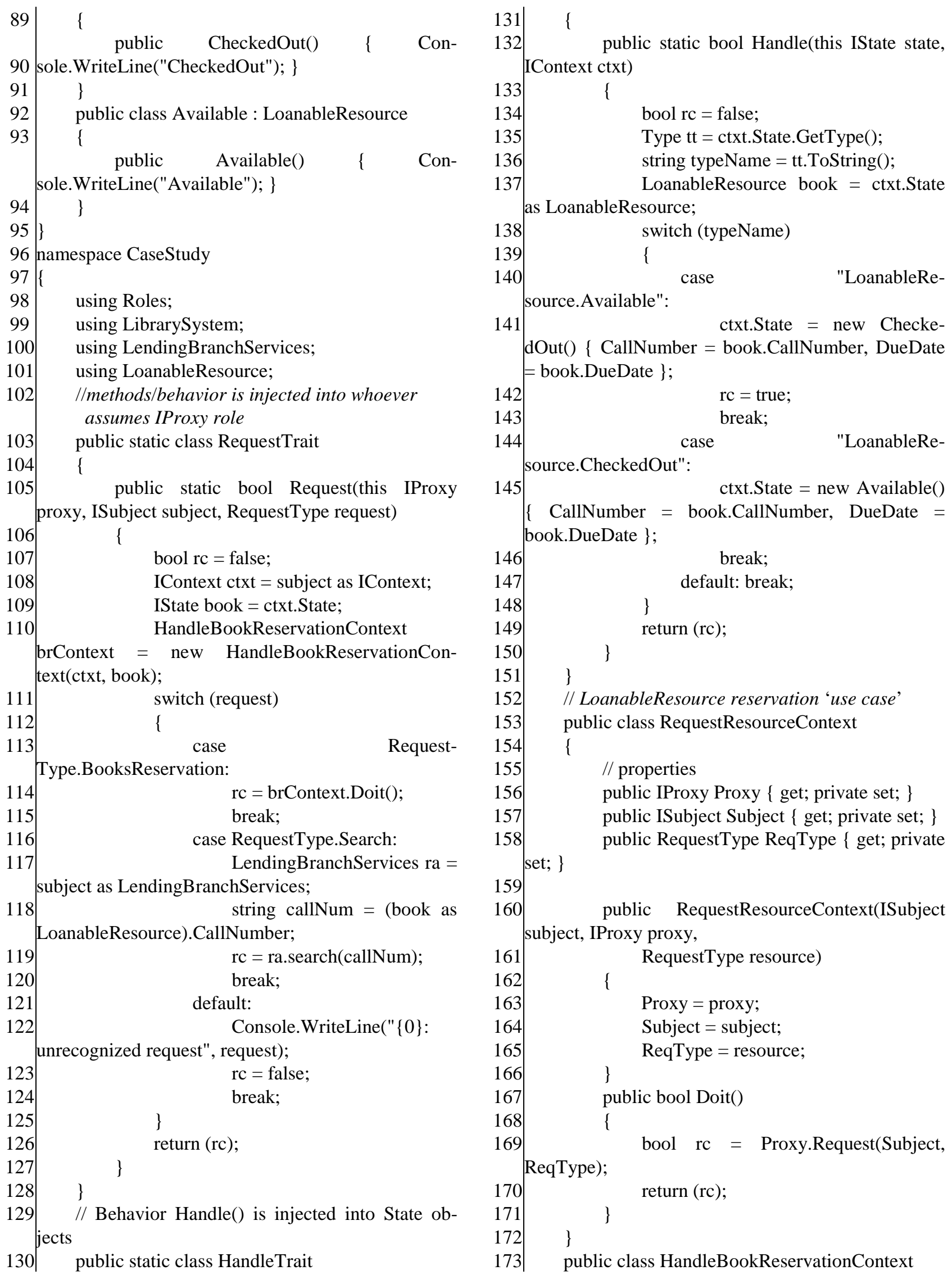




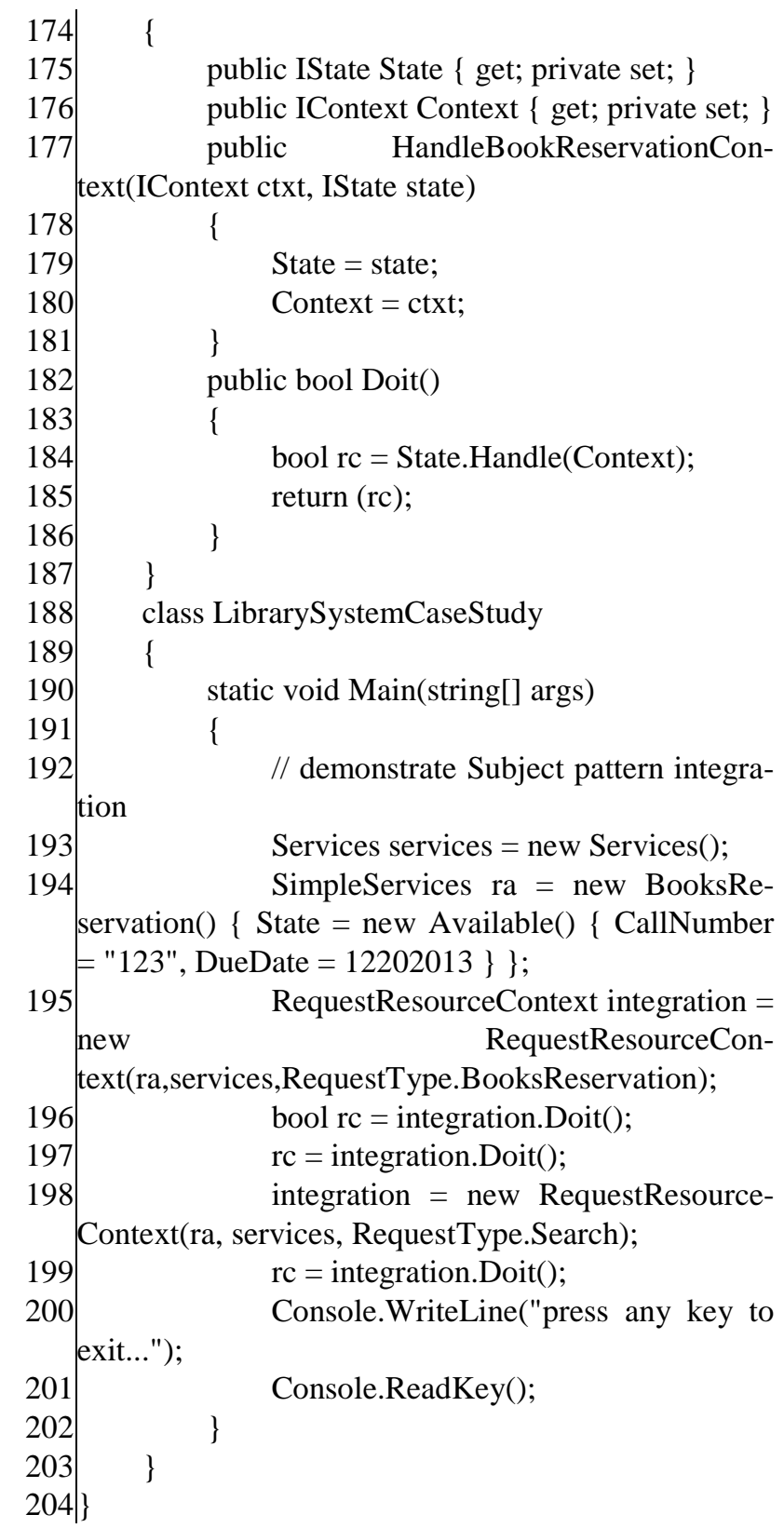

